Meta

Journal des traducteurs

Translators' Journal

\title{
Liste des relecteurs 2009
}

Volume 54, numéro 4, décembre 2009

URI : https://id.erudit.org/iderudit/044185ar

DOI : https://doi.org/10.7202/044185ar

Aller au sommaire du numéro

Éditeur(s)

Les Presses de l'Université de Montréal

ISSN

0026-0452 (imprimé)

1492-1421 (numérique)

Découvrir la revue

Citer ce document

(2009). Liste des relecteurs 2009. Meta, 54(4), 643-645.

https://doi.org/10.7202/044185ar

Ce document est protégé par la loi sur le droit d'auteur. L'utilisation des services d'Érudit (y compris la reproduction) est assujettie à sa politique d'utilisation que vous pouvez consulter en ligne.

https://apropos.erudit.org/fr/usagers/politique-dutilisation/
Cet article est diffusé et préservé par Érudit.

Érudit est un consortium interuniversitaire sans but lucratif composé de l’Université de Montréal, l'Université Laval et l'Université du Québec à Montréal. Il a pour mission la promotion et la valorisation de la recherche. https://www.erudit.org/fr/ 


\section{Liste des relecteurs 2009}

Nous remercions chaleureusement tous ceux et celles qui ont donné de leur temps et qui contribué à la revue. Toute omission est involontaire de notre part, veuillez nous la signaler.

May Abouzahra (Université Acadia)

Bassey Antia (University of Maiduguri)

Maite Aragonés Lumeras (WIPO, Genève)

Lourdes Arencibia (Universidad de la Habana)

Dimitris Asimakoulas (University of Surrey)

Fabienne Baider (Université de Chypre)

Michel Ballard (Université d'Artois)

Paul Bandia (Université Concordia)

Salah Basalamah (Université d'Ottawa)

Sabine Bastian (Université de Leipzig)

Nicole Baumgarten (University of Southern Denmark)

Elisabeth Bladh (Université de Stockholm)

Xavier Blanco (Universitat Autònoma de Barcelona)

Annie Brisset (Université d'Ottawa)

Laurent Brun (Université d'Ottawa)

Hélène Buzelin (Université de Montréal)

Leo Chan (Lingnan University)

Frederic Chaume Varela (Universitat Jaume I)

Mikyung Choi (Université Féminine d'Ewha)

Nicoleta Cinpoes (University of Worcester)

Luminita Ciumpe (Université de Montréal)

Kathleen Connors (Université de Montréal)

Sean Cotter (University of Texas in Dallas)

María Josep Cuenca (Universitat de València)

Jennifer De Camp (MITRE)

Raquel de Pedro Ricoy (Heriot-Watt University)

Akila Naïma Dib (Université de Montréal)

Jorge Díaz-Cintas (Imperial College London)

Joseph Dichy (Université Lumière - Lyon 2)

Valérie Dullion (École de traduction et d'interprétation - Université de Genève)

Keiran Dunne (Kent State University)

Álvaro Echeverri (Université de Montréal)

Rosa Estopà (Universitat Pompeu Fabra)

Daniel Gile (ESIT, Paris)

Nathalie Gormezano (ISIT, Paris) 
Jean-Marc Gouanvic (Université Concordia)

Mathieu Guidère (École de traduction et d'interprétation - Université de Genève)

Sandra Hale (University of Western Sydney)

Brenda Hosington (University of Warwick)

John Humbley (Université Paris Diderot - Paris 7)

María Gabriela Iturriza (Université de Montréal)

Gaston Gross (Université Paris 13)

Moira Inghilleri (University College London)

Hédi Jatlaoui (Université de Sousse)

Laurence Jay-Rayon (Université de Montréal)

Ronald Jenn (Université Charles-de-Gaulle - Lille 3)

Klaus Kaindl (University of Vienna)

Dorothy Kelly (Universidad de Granada)

Douglas Kibbee (University of Illinois)

Aurelia Klimkiewicz (Glendon College, York University)

Alexander Künzli (Zurich University of Applied Sciences)

Ingrid Kurz (University of Vienna)

Efi Lamprou (Université de Chypre

Gillian Lane-Mercier (Université McGill)

Christopher Larkosh (University of Massachusetts Dartmouth)

Elisabeth Lavault-Olléon (Université Stendhal - Grenoble 3)

Elisabeth Le (University of Alberta)

$\mathrm{Mu}$ Lei (Hainan University)

Kazem Lotfipour-Saedi (Université d'Ottawa)

Peter Low (University of Canterbury)

Rachel Lung (University of Hong Kong)

Rumyana Lyutakova (Sofia University)

Danièle Marcoux (Université Concordia)

Elizabeth Marshman (Université d'Ottawa)

Danny Masschelein (Hogeschool-Universiteit Brussel)

Ian Mason (Heriot-Watt University)

Alan Melby (Brigham Young University)

Ginette Michaud (Université de Montréal)

Robert Neather (Hong Kong Baptist University)

Alexis Nouss (Cardiff University)

Riitta Oittinen (University of Tampere)

Pilar Orero (Universitat Autónoma de Barcelona)

Jeannette Ørsted (CLS Communication AG, Bâle)

Béchir Ouerhani (Université de Sousse)

Thi Cuc Phuong Nguyen (Université de Hanoi)

Man-Ghyu Pak (Ajou University)

Réal Paquette (Université de Montréal)

Mayra Parra (Université de Montréal)

Richard Patry (Université de Montréal)

Franz Pöchhacker (University of Vienna)

Emily Poon Wai-Yee (Open University of Hong Kong)

Tom Priestly (University of Alberta) 
Jean Quirion (Université d'Ottawa)

Antin Fougner Rydning (Université d'Oslo)

Julio César Santoyo (Universidad de León)

Véronique Sauron (École de traduction et d'interprétation - Université de Genève)

Christina Schäffner (Aston University)

Christopher Scott-Tennent (Universitat Oberta de Catalunya)

Candace Séguinot (Glendon College, York University)

Wei Shao (Université des langues étrangères de Beijing)

Sherry Simon (Université Concordia)

Pamela J. Smith (University of Nebraska at Omaha)

Gérard Snow (Université de Moncton)

James St. André (The University of Manchester)

Min Sun (Université Laval)

Şehnaz Tahir-Gürçağlar (Bogazici University)

Carol Taylor Torsello (University of Padua)

Jan Van Coillie (Hogeschool-Universiteit Brussel)

Lawrence Venuti (Temple University)

Luise von Flotow (Université d'Ottawa)

Judy Wakabayashi (Kent State University)

Anthony Wall (University of Calgary)

Hui Wang (City University of Hong Kong)

Kefei Wang (Beijing Foreign Studies University)

Qianting Wang (China Radio International, Beijing)

Jane Wilhelm (Boston University Geneva Program)

Lia Wyler (Rio de Janeiro)

Yong Zhong (University of New South Wales)

Jehan Zitawi (Abu Dhabi University)

Jean-Philippe Zouogbo (Université Paris Diderot - Paris 7) 